\title{
A computerised system to evaluate patients with rheumatic diseases
}

\author{
Authors: Raashid Luqmani, Lorraine O'Neill and Joel David
}

\begin{abstract}
Aims
Management decisions for complex cases should be made on the basis of multidisciplinary consensus in order to ensure that the optimal treatment decisions are made within a setting of increasing case complexity coupled with an expanding range of treatment options, many of which are very expensive. An NHS England commissioning for quality and innovation (CQUIN) was introduced for autoimmune rheumatic diseases in 2016. The aim of this proposal was to develop and implement an innovative software system to capture data on clinical caseload including complex disease status assessment using validated measures so that we could evaluate each patient and use a multidisciplinary review process to define the optimal individualised care plan for them, thereby fulfilling the requirements of the CQUIN.
\end{abstract}

\section{Methods}

The software system was designed using Microsoft Access (version 2013) and consists of series of a main central form for diagnosis and treatment which is linked to series of sub-forms to document the assessment of each individual conditions using validated assessments. Use of the system was restricted in accordance with Caldicott guidelines. We undertook structured multidisciplinary meetings with a minimum of two consultants and a nurse specialist to discuss and document the rationale for treatment decisions.

\section{Results}

Between April 2016 and September 2017, 2,213 patient episodes were submitted for review in multidisciplinary teams (MDTs). Patients underwent formal evaluation of their disease status by the individual clinicians prior to the MDT review. Thirty-four per cent had giant cell arteritis, $11 \%$ had anti-neutrophil cytoplasm antibody associated vasculitis, $10.6 \%$ had systemic lupus erythematosus, $8 \%$ had myositis/interstitial lung disease and $6 \%$ had scleroderma/interstitial lung disease. The remainder had other forms of vasculitis or connective tissue disease. Table 1 summarises the main outcomes of the MDT meetings and Table 2 shows the detailed treatment changes recommended.

Author: Rheumatology, Oxford University Hospitals NHS Foundation Trust, Oxford, UK

\begin{tabular}{|c|c|}
\hline $\begin{array}{l}\text { Outcomes from multidisciplinary team meetings for } \\
2,213 \text { cases }\end{array}$ & $\%$ \\
\hline Major changes in therapy & 39 \\
\hline Minor changes in therapy & 18.7 \\
\hline No change in therapy & 42 \\
\hline Further investigations & 36 \\
\hline Referrals to other specialists & 7.5 \\
\hline
\end{tabular}

Table 2. Detailed treatment changes recommended

Details of major therapy changes as a result of multidisciplinary team meetings for 2,213 cases

Change in steroid treatment 16.5

Change in disease-modifying therapy 18.8

Cessation of disease-modifying therapy 3.8

Start a biologic agent 1.8

Continue a biologic agent $\quad 0.7$

Stop a biologic agent

Note: Although a variety of biologic agents were used, the most commonly prescribed within this cohort was rituximab.

In over a third of cases, a major change in therapy was agreed; further investigations were recommended in $36 \%$ of cases and further referrals to other specialists in $7.5 \%$. A low proportion of these cases were recommended to start a biologic agent (1.8\%) or continue it $(0.7 \%)$, while the majority were managed with lessexpensive, conventional treatments such as cyclophosphamide or azathioprine. Without the MDT process, we predict that at least $10 \%$ of these patients would have been prescribed a biologic agent.

\section{Conclusion}

We have designed and implemented an innovative unified data collection system for a range of complex inflammatory diseases which potentially require use of high cost drugs. We have used the system as the basis for creating quarterly reports to specialised commissioners defining our caseload, severity 
of disease and use of high and low cost drugs according to approved pathways. We have been able to produce individualised treatment plans ratified by the MDT, to justify prescribing decisions based on fulfilment of criteria defined in the National Institute for Health and Care Excellence and NHS England guidance. The system could be implemented more widely to other hospitals and also expanded to other specialist services requiring evaluation of complex cases.

\section{Conflict of interest statement}

None declared. 